\title{
Comparison of different types of endovascular mechanical embolectomy in acute ischemic stroke
}

Yuan Pu

1. Department of Neurosurgery, Suqian People' hospital of Nanjing Durm Tower hospital Group, Suqian, Jiangsu, China

\begin{abstract}
SUMMARY
Background: To compare the treatment efficacy of different types of endovascular mechanical embolectomy in acute ischemic stroke (AIS). Material and Methods: A total of 89 patients with AIS were selected in our hospital from January 2014 to January 2016 and divided into tPA group ( $n=27)$, tPA+Trevo group $(n=30)$ and $t P A+S o l i t a i r e ~ F R ~ g r o u p ~(n=32)$ for different treatments. Treatment effectiveness was evaluated using NIHSS and mRS system. The NIHSS score, vascular recanalization rate and postoperative complications were compared among groups. Results: The NIHSS score of the tPA group was significantly lower than that of other two groups at $1 d$ after the operation $(p<0.05)$, but it was significantly higher than that of other two groups at $3 d$ and $3 w$ after the operation ( $p<$ 0.05). After the treatment, no significant difference in NIHSS score was found between the tPA+Trevo and tPA Solitaire FR groups. The revascularization rate was significantly higher, but the mortality rate in $90 \mathrm{~d}$ was significantly lower in the $t P A+T r e v o$ and $t P A+S o l i t a i r e$ FR groups than that in the tPA group $(p<0.05)$, and no significant difference was found between the tPA+Trevo and tPA+Solitaire FR groups. The incidence rate of symptomatic intracranial hemorrhage was significantly lower in the tPA+Solitaire FR group than that in $T P A+$ Trevo group $(p<0.05)$ or TPA group $(p<0.01)$. Significantly more patients with $m R S$ no higher than 2 points were found in the tPA+Trevo and $t P A+$ Solitaire FR groups than those in tPA group $(p<0.05)$, and no significant difference was found between the tPA+Trevo and tPA+Solitaire FR groups. Conclusion: TPA+Solitaire FR is a type of thrombectomy that is superior to tPA and tPA+Trevo in the treatment of patients with AIS.
\end{abstract}

Keywords: Stroke. Embolectomy. Tissue plasminogen activator.

\section{INTRODUCTION}

A cerebrovascular accident (CVA), also called stroke, is a serious disease that ranks in second place among all death-causing diseases ${ }^{1}$. Besides death, stroke can also cause disability, which in turn seriously affects the patients' quality of life and brings heavy economic burden to both patients and their families ${ }^{2-4}$. According to the pathogenesis, stroke can be divided into subtypes including hemorrhagic stroke and ischemic stroke. As the dominant type of stroke, ischemic stroke accounts for about $80 \%$ of all stroke cases ${ }^{5-7}$. An ischemic stroke is caused by insufficient oxygen and blood supply to the brain, which can be caused by a variety of reasons, and in turn leads to brain tissue necrosis. As a type of ischemic stroke, acute ischemic stroke (AIS) is characterized by the sudden loss of blood supply to brain tissues $^{8}$. Clinical studies have proven that thrombotic or embolic occlusion is the main cause of most $\mathrm{AIS}^{9}$. 
Therefore thrombolysis has become a central part of the AIS treatment.

Intravenous recombinant tissue plasminogen activator, or tPA, has been recognized as the standard treatment for AIS. But no more than half of the patients showed complete recovery after the treatments with tPA within $4.5 \mathrm{~h}$ of stroke onset, and the motility rate is high [10]. The application of tPA treatment is also challenged by the existence of contraindications, and only less than $10 \%$ of AIS patients can be treated with it. In addition, tPA treatment usually fails to provide satisfactory outcomes for occlusion in main artery. The combined use of tPA and mechanical thrombectomy devices has been shown to be able to significantly improve the recanalization rate and clinical outcome. Solitaire FR stent and Trevo stent are two mostly used thrombectomy devices. However, the comparison of treatment efficacy and safety of those two systems still hasn't been reported.

In this study, a total of 89 patients with AIS were selected to be treated with different measures. The treatment outcomes and safety of those treatments were compared.

\section{MATERIALS AND METHODS}

\section{Patients}

A total of 89 patients with AIS enrolled from January 2014 to January 2016 in the Suqian People' hospital of Nanjing Durm Tower hospital Group were selected. Patients were divided into tPA group $(\mathrm{n}=27)$, tPA+Trevo group $(\mathrm{n}=30)$ and $\mathrm{tPA}+$ Solitaire FR group $(n=32)$ for different treatments according to their willingness and the physician's recommendations. Inclusion criteria: (1) Patients met the diagnostic criteria of AIS in China; (2) Patients diagnosed by imaging and biochemical examinations. Exclusion criteria: (1) patients with severe heart, liver, and kidney dysfunction; (2) patients who were not willing to receive treatment.

\section{Treatment}

Patients in the tPA group were treated with emergent intravenous thrombolysis: patients were treated with recombinant tissue plasminogen activator (rt-PA) alteplase at a dose of $0.9 \mathrm{mg} / \mathrm{kg}$ through intravenous injection (the first $10 \%$ ) and continuous intravenous pumping for $1 \mathrm{~h}$. The treatment was stopped if patients showed severe headache, hypertension, and nausea or vomiting. Patients in the TAP+Solitaire
FR group were treated with emergent intravenous thrombolysis combined with mechanical thrombectomy with solitaire FR stent: whole-brain CT digital subtraction angiography was performed to identify the cerebral arterial occlusion site and responsible vessels. A micro-guidewire was pushed through the thrombus occlusion site under the guidance of a path map. The stent delivery catheter was introduced to go through the micro-guidewire and pass the distal end of occlusion, and the Solitaire FR stent was introduced to go through the catheter. The Solitaire FR stent was opened and the opening state was maintained for 2-3 min after the recovery of local blood flow. This operation can be repeated no more than 3 times. Imaging examination was performed to evaluate the treatment efficacy. Patients in the tAP+Trevo group were treated with emergent intravenous thrombolysis that combined mechanical thrombectomy with the Trevo stent using the same method described above.

\section{Observation indicators}

Treatment effectiveness was evaluated using the NIHSS system at $1 \mathrm{~d}, 3 \mathrm{~d}$, and $3 \mathrm{w}$ before and after the treatment. A lower score indicates a better outcome. The vascular recanalization rate and symptomatic intracranial hemorrhage rate were recorded. Treatment outcomes were also evaluated using modified Rankin Scale (mRS), and patients with mRS score $\leq$ 2 were recorded.

\section{Statistical analysis}

SPSS19.0 software was used for all of the statistical analyses. Comparisons between groups were performed by t-test or chi-square test and $\mathrm{p}<0.05$ was considered to be statistically significant.

\section{RESULTS}

Comparison of general information between groups

No significant differences in age, gender, weight, cultural level, disease types and the sites of intracranial occlusion were found among three groups of patients (Table 1).

Comparison of imaging results before and after mechanical embolectomy

Using a patient from the tAP+Trevo group as an example, whole cerebral angiography showed oc- 
TABLE1 COMPARISON OF GENERAL INFORMATION AMONG GROUPS

\begin{tabular}{|c|c|c|c|c|}
\hline & $\begin{array}{l}\mathrm{tPA} \\
\mathrm{n}=27\end{array}$ & $\begin{array}{l}\mathrm{tPA}+\text { Trevo } \\
\mathrm{n}=30\end{array}$ & $\begin{array}{l}\mathrm{tPA}+\text { Solita } \\
\mathrm{n}=32\end{array}$ & \\
\hline Age & $65.17(5.84)$ & $68.87(7.69)$ & $63.87(4.13)$ & $p>0.05$ \\
\hline \multirow[t]{3}{*}{ Gender } & & & & $p>0.05$ \\
\hline & Male & 15 & 17 & 17 \\
\hline & Female & 12 & 13 & 15 \\
\hline Weight & $54.81(7.85)$ & $55.43(6.46)$ & $56.43(5.87)$ & $p>0.05$ \\
\hline \multirow[t]{4}{*}{ Cultural level } & & & & $p>0.05$ \\
\hline & Junior high school & 7 & 9 & 11 \\
\hline & Senior high school & 10 & 11 & 12 \\
\hline & College degree and above & 10 & 10 & 9 \\
\hline \multirow[t]{4}{*}{ Type of disease } & & & & $p>0.05$ \\
\hline & Hypertension & 16 & 20 & 19 \\
\hline & Diabetes & 6 & 6 & 8 \\
\hline & Atrial fibrillation & 5 & 4 & 5 \\
\hline Site of intracranial occlusion & & & & $p>0.05$ \\
\hline Carotid $T$ & 6 & 6 & 8 & \\
\hline Proximal M1 & 9 & 11 & 11 & \\
\hline Distal M1 & 5 & 4 & 5 & \\
\hline M2 & & 3 & 5 & 3 \\
\hline Basilar artery & 2 & 1 & 2 & \\
\hline Proximal ICA stenosis $>70 \%$ & 2 & 3 & 3 & \\
\hline
\end{tabular}

A

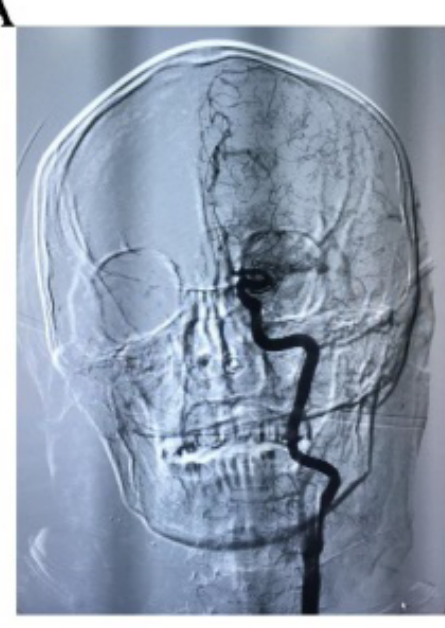

C

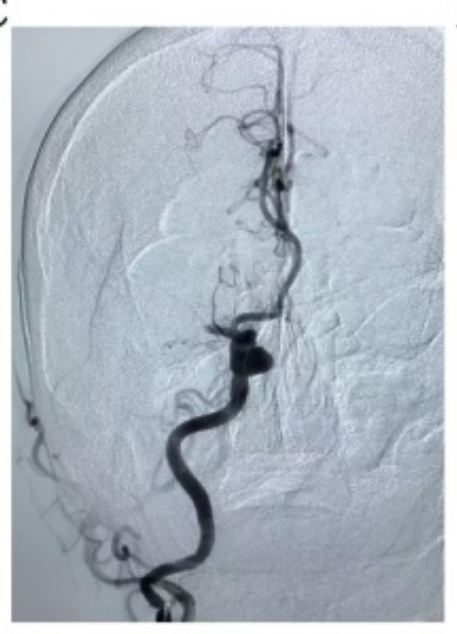

B

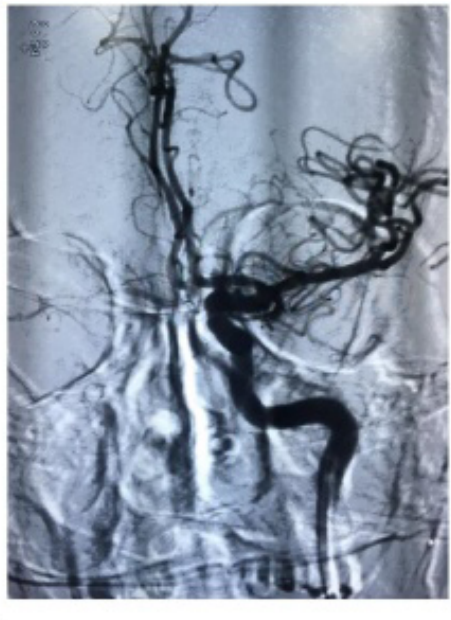

D

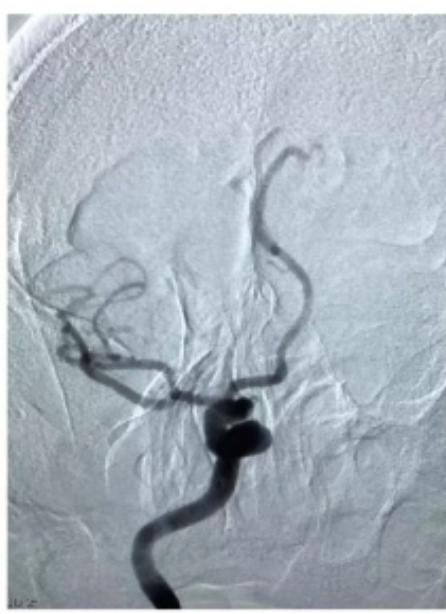

FIGURE 1 COMPARISON OF IMAGING RESULTS BEFORE AND AFTER MECHANICAL EMBOLECTOMY

A Whole cerebral angiography showed occlusion at the distal end of the M1 section of the left middle cerebral artery. B The occlusion was removed after the operation. CWhole cerebral angiography showed occlusion at the beginning site of the right internal carotid artery. D The vessel was opened after operation. E Thrombus collected after operation.

E

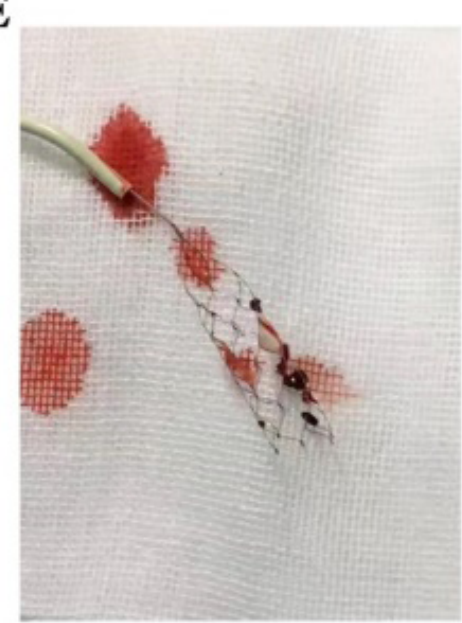


TABLE 2. COMPARISON OF TREATMENT OUTCOMES

\begin{tabular}{|c|c|c|c|c|c|}
\hline & No. & $\mathrm{TICl}$ & $\mathrm{SICH}$ & Mortality $90 \mathrm{D}(\%)$ & $m R S \leq 2$ \\
\hline tPA & 27 & $16(59.26)$ & 3(10.69) & $6(22.22)$ & $9(33.33)$ \\
\hline tPA + Trevo & 30 & $22(73.30)$ & $2(6.67)$ & $5(16.67)$ & $17(56.67)$ \\
\hline tPA + Solitaire FR & 32 & $26(81.25)$ & $1(3.125)$ & $4(12.50)$ & $20(62.50)$ \\
\hline p value $e^{\mathbf{a}}$ & $<0.05$ & $>0.05$ & $>0.05$ & $<0.05$ & \\
\hline p value ${ }^{\text {b }}$ & $<0.05$ & $<0.05$ & $<0.05$ & $<0.05$ & \\
\hline p value & $>0.05$ & $<0.01$ & $>0.05$ & $>0.05$ & \\
\hline
\end{tabular}

Notes: $\mathbf{a}$, comparison between the tPA+Trevo and tPA groups; $\mathbf{b}$, comparison between the tPA+ Solitaire FR and tPA groups; $\mathbf{c}$, comparison between the tPA + Trevo and tPA+Solitaire FR groups; TICI, Thrombolysis In Cerebral Infarction; SICH, symptomatic intracranial hemorrhage.

clusion at the distal end of the M1 section of the left middle cerebral artery (Fig. 1A); the occlusion was removed after mechanical thrombectomy with Trevo stent (Fig. 1B). Using a patient from the tAP+Trevo group as another example, whole cerebral angiography showed occlusion at the beginning site of the right internal carotid artery (Fig. 1C), and the vessel was opened after mechanical thrombectomy with Solitaire FR stent (Fig. 1D).

Comparison of NIHSS scores among three groups before and after the treatment

There was no significant difference in NIHSS scores among three groups before the treatment. The NIHSS score of the tPA group was significantly lower than that of other two groups at $1 \mathrm{~d}$ after the operation $(p<0.05)$, but it was significantly higher than that of other two groups at $3 \mathrm{~d}$ and $3 \mathrm{w}$ after the operation $(p<0.05)$. No significant difference in NIHSS scores was found between the tPA+Trevo and tPA Solitaire FR groups after the operation.

\section{Comparison of treatment outcomes among three groups}

The revascularization rate of the $\mathrm{AP}+$ Trevo and tPA+Solitaire FR groups was significantly higher than that of the tPA group $(p<0.05)$, but no significant difference was found between the tPA+Trevo and tPA+Solitaire FR group. The incidence rate of symptomatic intracranial hemorrhage was significantly lower in the TPA+Solitaire FR group than that in the tPA+Trevo $(p<0.05)$ and tPA groups $(p<0.01)$, and no significant difference was found between the tPA+Trevo and tPA groups. The mortality rate at 90 $\mathrm{d}$ was lower in the tPA+Trevo and tPA+Solitaire FR groups than that in TPA groups, but significant difference was only found between the tPA+Solitaire FR and tPA groups $(p<0.05)$. The number of patients with mRS no higher than 2 points is significantly bigger in the tPA+Trevo and tPA+Solitaire FR groups than in the tPA group $(p<0.05)$, but no significant difference was found between the tPA+Trevo and tPA+Solitaire FR groups (Table 2).

\section{DISCUSSION}

AIS is a serious disease that causes unacceptably high morbidity and mortality rates worldwide. Treatment with tPA is the standard for AIS patients. However, the treatment with tPA alone usually fails to provide satisfactory outcomes due to the short treatment time window, which is only 3 to 4.5 hours. Therefore, how to extend this time window has become a focus of the AIS treatment. A previous study reported that the combined use of tPA and 3-methyl1-phenyl-2-pyrazoline-5-one (a free radical scavenger) could significantly extend the ischemic therapeutic time, which in turn would increase survival rates and reduced the incidence of postoperative complications. In another study, Fisher and Albers found that the application of tPA under the guidance of advanced imaging can also extend the therapeutic time window of AIS. Although those newly developed techniques showed promising outcomes in the AIS treatment in comparison with tPA alone, all those treatments may fail to increase the revascularization rate, especially for occlusions in main artery due to the lack of endovascular treatment.

In recent years, endovascular mechanical embolectomy combined with tPA treatment has been widely used in the treatment of AIS, and it showed high efficiency in enhancing revascularization as well as expanding the treatment time window. A series of mechanical thrombectomy devices and stent retrievers have been developed. Since these are the two most commonly used mechanical thrombecto- 
my devices, more and more patients have accepted the application of Solitaire FR stent and Trevo stent. As a kind of clot retrieval, Solitaire Flow stent contains a self-expanding stent retriever that can remove proximal vessel occlusion in patients with AIS to restore blood flow. A prospective study carried out by Pereira et al. found that the treatment with Solitaire Flow stent could significantly improve clinical outcomes and reduce postoperative complications. Another type of clot retrieval, Trevo works in a similar way to that of Solitaire FR stent. In an animal model, Trevo was proved to be effective in inducing reperfusion of blocked arteries without leading to significant disruption of the vascular integrity.

To date, the comparison of the treatment efficacy and safety of Solitaire FR as well as Trevo has not been well studied although there was one relevant study by Mendonça et al. In that study, they reported that the revascularization rate of patients who received Solitaire Stent treatment (60 \%) is lower than that of patients in the Trevo group (77\%), but the difference was not significant. The incidence rate of postoperative symptomatic intracranial hemorrhage was lower in the Trevo group $(0 \%)$ than that in Solitaire Stent group (15\%). In addition, no significant difference in mortality rate after 3 months was found between the Trevo stent and Solitaire Stent groups. Consistent with this study, in our study, no significant difference in revascularization rate was found between the tPA+Trevo and tPA+Solitaire FR groups, but the revascularization rate of $\mathrm{TP}+\mathrm{Trevo}$ and tPA+Solitaire FR groups was significantly higher than that of tPA group. In addition, mortality rate at $90 \mathrm{~d}$ was lower in $\mathrm{TPA}+$ Trevo and $\mathrm{tPA}+$ Solitaire FR groups than in the tPA group. Those results suggest that, compared with the use of tPA alone, the combined use of Trevo stent and tPA+Solitaire stent can significantly increase the revascularization rate and reduce the mortality rate. Different results were also found in our study. In this study, the incidence rate of symptomatic intracranial hemorrhage was significantly lower in the tPA+Solitaire FR group than that in tPA+Trevo and tPA groups, but no significant difference was found between the tPA+Trevo and tPA groups. This result suggests that, compared with the use of Trevo stent, the Solitaire FR can significantly reduce the incidence of symptomatic intracranial hemorrhage.

In this study, NIHSS and mRS scoring systems were used to evaluate the treatment outcomes as well as the safety of different treatments. The NIHSS score of the tPA group was significantly lower than that of other two groups at $1 \mathrm{~d}$ after the operation, but it was significantly higher than that of other two groups at $3 \mathrm{~d}$ and $3 \mathrm{w}$ after the operation, indicating that the use of Solitaire and Trevo could only improve the medium- and long-term treatment outcomes but not the short-term outcomes. In addition, a number of patients with mRS that no higher than 2 points is significantly bigger in the tPA+Trevo group and tPA+Solitaire FR groups than in the tPA group, which further confirmed that advantages of the combined treatment.

\section{CONCLUSION}

In conclusion, the effectiveness and safety of tPA+Trevo and tPA+Solitaire in the treatment of AIS patients were better than those of tPA alone. $\mathrm{PA}+\mathrm{Sol}-$ itaire is also superior to tPA+Trevo in some aspects. This study is limited by the small sample size. Further studies with bigger sample size are needed to confirm the conclusions of this study.

\section{Acknowledgments}

We thank the financial support from Science and Technology Support Plan Project from Suqian City (No. S201613).

\section{RESUMO}

OBJETIVO Comparar a eficácia do tratamento de diferentes tipos de embolectomia mecânica endovascular em acidente vascular cerebral isquêmico agudo (AIS).

MATERIAL E MÉTODOS Um total de 89 pacientes com AIS foi selecionado em nosso hospital de janeiro de 2014 a janeiro de 2016 , e os pacientes foram divididos em: grupo tPA $(n=27)$, tPA + grupo Trevo $(n=30)$ e grupo tPA + Solitaire FR $(n=32)$ para diferentes tratamentos. A eficácia do tratamento foi avaliada usando NIHSS e sistema mRS. Escore NIHSS, taxa de recanalização vascular e complicações pós-operatórias foram comparados entre os grupos.

RESULTADOS A pontuação NIHSS do grupo tPA foi significativamente menor do que a dos outros dois grupos em um dia após a operação $(p<0,05)$, mas foi significativamente maior do que nos outros dois grupos em três dias e três semanas após a operação $(p<0,05)$. Após o tratamento, não houve diferença significativa no escore NIHSS entre o grupo tPA + Trevo e o gru- 
po tPA Solitaire FR. A taxa de revascularização foi significativamente maior, mas a taxa de mortalidade em 90 dias foi significativamente menor nos grupos tPA + Trevo e tPA + Solitaire FR do que no grupo tPA $(p<0,05)$ e não houve diferença significativa entre os grupos tPA + Trevo e tPA + Solitaire FR. A taxa de incidência de hemorragia intracraniana sintomática foi significativamente menor no grupo tPA + Solitaire FR do que no grupo tPA + Trevo $(p<0,05)$ ou no grupo tPA $(p<0,01)$. Significativamente mais pacientes com mRS não maiores que 2 pontos foram encontrados no grupo tPA + Trevo e tPA + Solitaire FR do que no grupo tPA $(p<0,05)$, e nenhuma diferença significativa foi encontrada entre os grupos tPA + Trevo e tPA + Solitaire FR. Conclusão O tPA + Solitaire FR é um tipo de trombectomia superior ao tPA e tPA + Trevo no tratamento de pacientes com EIA. PALAVRAS-CHAVE: Acidente vascular cerebral. Embolectomia. Ativador de plasminogênio tecidual.

\section{REFERENCES}

1. Yang J, Yin P, Zhou M, Ou CQ, Li M, Li J, et al. The burden of stroke mortality attributable to cold and hot ambient temperatures: epidemiological evidence from China. Environ Int. 2016;92-93:232-8.

2. Park JS, Hwang NK, Oh DH, Chang MY. Effect of head lift exercise on kinematic motion of the hyolaryngeal complex and aspiration in patients with dysphagic stroke. J Oral Rehabil. 2016;44(5):385-91.

3. Keppel Hesselink JM. NS1209/SPD 502, a novel selective AMPA antagonist for stroke, neuropathic pain or epilepsy? Drug development lessons learned. Drug Dev Res. 2017;78(2):75-80.

4. Seto SW, Chang D, Jenkins A, Bensoussan A, Kiat H. Angiogenesis in ischemic stroke and angiogenic effects of Chinese herbal medicine. J Clin Med. 2016;5(6). pii: E56.

5. Vasileva D, Lubenova D, Mihova M, Grigorova-Petrova K, Dimitrova A. Orthostatic reactivity in patients with ischemic stroke in the chronic period. Open Access Maced J Med Sci. 2015;3(3):397-402.

6. Boisserand LS, Kodama T, Papassin J, Auzely R, Moisan A, Rome C, et al. Biomaterial applications in cell-based therapy in experimental stroke. Stem Cells Int. 2016;2016:6810562.
7. Liang Y, Huang J, Tian J, Cao Y, Zhang G, Wang C, et al. The prevalence and risk factors of stroke in patients with chronic schizophrenia. Neuropsychiatr Dis Treat. 2016;12:1131-4.

8. Powers WJ, Derdeyn CP, Biller |, Coffey CS, Hoh BL, Jauch EC, et al; American Heart Association Stroke Council. 2015 American Heart Association/ American Stroke Association Focused Update of the 2013 guidelines for the early management of patients with acute ischemic stroke regarding endovascular treatment: a guideline for healthcare professionals from the American Heart Association/American Stroke Association. Stroke. 2015;46(10):3020-35.

9. Chimowitz MI. Endovascular treatment for acute ischemic stroke: still unproven. N Engl J Med. 2013;368(10):952-5.

10. Wardlaw IM, Murray V, Berge E, del Zoppo G, Sandercock P, Lindley $R L$, et al. Recombinant tissue plasminogen activator for acute ischaemic stroke: an updated systematic review and meta-analysis. Lancet. 2012;379(9834):2364-72. 